\title{
Pathogenic Microorganisms and Pancreatic Cancer
}

\author{
Chunsaier Wang Jingnan Li \\ Department of Gastroenterology, Peking Union Medical College Hospital, Beijing, China
}

\section{Key Words}

Bacteria $\cdot$ Chronic hepatitis virus infection $\cdot$ Helicobacter pylori $\cdot$ Microbiome $\cdot$ Oral microbiota Pancreatic cancer

\begin{abstract}
Background: Pancreatic cancer is one of the most lethal cancers worldwide. No effective screening methods exist, and available treatment modalities do not effectively treat the disease. Established risk factors for pancreatic cancer, including smoking, chronic pancreatitis, obesity and type 2 diabetes mellitus, collectively account for less than half of all pancreatic cancer cases. Accumulating reports have demonstrated that there is an association between pathogenic microorganisms and pancreatic cancer. Summary: A substantial amount of preclinical and clinical evidence suggests that microbiota are likely to influence pancreatic carcinogenesis. This review summarizes the literature on studies examining infections that have been linked to pancreatic cancer. Key Message: Helicobacter pylori infection may be a risk factor for pancreatic cancer; chronic hepatitis virus and oral microbiota may also play a role in pancreatic carcinogenesis. Practical Implications: Considering the worldwide burden of the disease, the association between microbiota and pancreatic cancer in this review may provide new ideas to prevent and treat pancreatic cancer more efficiently. Further studies in this direction are urgently needed.

(C) 2015 S. Karger AG, Basel
\end{abstract}

\section{Introduction}

Each year, approximately 40,000 Americans die of pancreatic cancer, making it the fourth most common cause of cancer-related mortality [1]. The survival time of pancreatic cancer is very short, with fewer than half of patients surviving past 6 months from diagnosis. There is 
no effective screening method, and consequently primary prevention of pancreatic cancer is of particular importance in reducing the burden of this malignancy. Except for the established risk factors for pancreatic cancer, including smoking, chronic pancreatitis, obesity and type 2 diabetes mellitus (T2DM), pathogenic microorganisms also contribute to the carcinogenesis and progression of pancreatic cancer. In this review, we summarize the literature on the relationship between pathogenic microorganisms and pancreatic cancer.

\section{Gut Microorganisms and Relative Disease}

More than $10^{14}$ microorganisms, which represent up to 1,150 different species and a total genome comprising 150-fold more genes than the human genome, live in our gastrointestinal tract (GIT) [2]. These microbiota form a virtual bioreactor facilitating digestion, nutrient provision and the shaping of our immune system [3]. As the gut microbiota appear to contribute to nearly every aspect of the host's growth and development, it is not surprising that a tremendous array of diseases and dysfunctions have been associated with an imbalance in either composition, numbers or habitat of the gut microbiota [4]. Disturbance in the gut microbial communities, created by the administration of antibiotics and adversely affecting the function of multiple host organ systems, has been recognized a time ago. One of the bestknown diseases is antibiotic-associated diarrhea, which can be due to the pathological overgrowth of Clostridium difficile in the antibiotic-treated GIT [5]. Also the increased reduction of gut microbial diversity concurrent with overgrowth of enterococci in critically ill patients following treatment in an intensive care unit was shown to correlate with greater organ failure and mortality [6]. Irritable bowel syndrome, a disorder of the GIT with complex etiology, sometimes develops following recovery from enteric infections [7], suggesting that infection-mediated disturbance in the host's gut microbial community results in GIT malfunction. As for inflammatory bowel diseases, evidence points to the intestinal microbiome being a key player in the development and perpetuation of the disease [8]. It has been tested that defects in the innate immune response to commensal intestinal bacteria, resulting in an exaggerated adaptive immune response to these organisms, are implicated in the pathogenesis of Crohn's disease [9]. Numerous attempts have been made to treat and/or prevent inflammatory bowel diseases through the use of pre- and/or probiotics, aiming to redress the inflammation-promoting imbalance in gut microbiota.

While the gut microbiota are an essential partner in health, some aspects of their presence can induce highly unwanted carcinogenic processes in the host. Perhaps the moststudied example of a microbiota-induced GIT malignancy is Helicobacter pylori-mediated gastric carcinoma [10]. In the last few years, several studies have presented tangential evidence that suggests a possible role of microbes in the pathogenesis of pancreatic cancer [11]. There are also some disorders of the GIT accessory organs, such as spontaneous bacterial peritonitis and hepatic encephalopathy [12], in whose pathogenesis luminal microbiota have been reported to play an important role. Furthermore, there is some research aiming to demonstrate the relationship between gut microorganisms and other systemic diseases, such as obesity, allergy, type 1 diabetes mellitus and even autism [13-16].

\section{Epidemiology and Risk Factors of Pancreatic Cancer}

The global annual incidence rate for pancreatic cancer is about 8/100,000 persons [17]. Adenocarcinoma is the most frequent type of pancreatic cancer. Incidence rates of pancreatic cancer vary among countries, with approximate 5- to 7-fold differences between countries 
with the lowest and highest incidence. Fewer than $10 \%$ of pancreatic cancer cases occur among individuals younger than 55 years old, and the median age at onset is 71 years; men have higher incidence rates than women [18]. In China, from 1998 to 2007, the annual incidence for men and women in urban areas showed an increase of 1.86 and $2.1 \%$ per year, but the increasing trend on the age-standardized rate was not obvious in either men or women. However, the incidence rates for men and women in rural areas increased by 7.54 and $7.83 \%$ and the age-standardized rates increased by 4.82 and $5.48 \%$ per year [19].

Previous epidemiological investigations have identified some possible risk factors of pancreatic cancer, such as smoking, chronic pancreatitis, alcohol, long-standing diabetes, African-American race, obesity and consumption of smoked or processed meat [17, 20, 21]. Genetic mutations, hereditary syndromes and familial aggregation may account for approximately $5-10 \%$ of cases [17]. Tobacco is the best-established risk factor for pancreatic cancer. In a comprehensive meta-analysis of 47 case-control and 35 cohort studies, Iodice et al. [22] calculated the respective summary risk estimates for current and former smokers to be 1.7 and 1.2. In the past, consuming alcohol was not considered an important risk factor for pancreatic cancer; however, recent studies have shown that daily consumption of $30 \mathrm{~g}$ of alcohol, or the equivalent of $>3$ glasses of any alcoholic beverage per day, is associated with a $20 \%$ increased risk of pancreatic cancer [21]. In China, a recent study showed that smoking, diabetes mellitus, hypertension, biliary disease and serum triglyceride level may be independent risk factors for pancreatic cancer [23]. Of all the risk factors, we focus on diabetes and obesity, which are related to the gut microbiota.

\section{Diabetes and Gut Microbiota}

T2DM is believed to be caused by a series of risk factors such as genetic liability, age, overweight or obesity as well as an unhealthy lifestyle. Recently, data have accumulated in animal models and humans, suggesting that T2DM is associated with a profound dysbiosis. A previously reported smaller study showed that the microbiota from T2DM differed from nondiabetic adults, showing that certain Lactobacillus species may be increased in T2DM [24]. In a later landmark study, high-throughput sequencing performed using stool samples from Chinese patients with T2DM and metagenomic analysis combined with clinical data showed that patients with T2DM exhibited a moderate intestinal dysbiosis characterized especially by a decrease in butyrate-producing Roseburia intestinalis and Faecalibacterium prausnitzii [25]. Intestinal microbiota may play an important role in the pathogenesis of T2DM by influencing body weight, bile acid metabolism, proinflammatory activity and insulin resistance as well as modulation of gut hormones [26].

\section{Obesity and Gut Microbiota}

The prevalence of obesity is increasing worldwide. In addition to the imbalance between energy intake and expenditure, sedentary lifestyle, a diet high in saturated fats and sugars and genetic predisposition, many other factors may be involved in obesity. Accumulating evidence indicates that the bacterial species of the gut play a significant role in the development of obesity [27]. Analysis of the fecal microbiota of morbidly obese subjects before and after Rou-en-Y gastric bypass surgery reported lower numbers of the Bacteroides-Prevotella group in obese subjects than in control subjects at baseline, while the numbers increased after surgery [28]. Another independent study, including normal-weight, obese and anorexic subjects, also reported lower Bacteroidetes gene copy numbers in obese human subjects compared to control subjects [29]. What's more, a recent study found a decrease in specific species (Bifidobacterium animalis and Methanobrevibacter smithii) and an increase in others, such as Staphylococcus aureus, Escherichia coli and Lactobacillus reuteri, which have been associated with obesity [30]. The mechanisms rely either on the 'energy harvest' hypothesis 
(an increased utilization of indigestible carbohydrates by microbial enzymes) or on the impact of microbial metabolites or cell-derived signals on the host pathways that regulate energy intake and/or fat deposition [31].

\section{Helicobacter pylori Infection and Pancreatic Cancer}

H. pylori, the ubiquitous bacterium that colonizes the human stomach, has been the subject of increased attention in the last 30 years. Over half of the modern human population is infected with $\mathrm{H}$. pylori, and its prevalence varies from 60-90\% in Japan, China, Russia and most of Central and Eastern Europe to 30-40\% in Western Europe and the United States [32]. Previous studies have examined the association between $H$. pylori infection and pancreatic carcinoma, but with inconsistent results. The Alpha-Tocopherol, Beta-Carotene Cancer Prevention (ATBC) Study, a prospective cohort study of male smokers, reported subjects positive for $H$. pylori antibodies or CagA-positive $H$. pylori strains to be at increased risk of developing pancreatic cancer (odds ratio [OR] = 1.87, 95\% confidence interval [CI] 1.05-3.34; OR $=2.01,95 \%$ CI 1.09-3.70, respectively) [33]. However, a case-control study in a Polish population reported that neither H. pylori $(\mathrm{OR}=1.27,95 \% \mathrm{CI} 0.64-2.61)$ nor CagA $(\mathrm{OR}=0.90$, 95\% CI 0.46-1.73) seropositivity were significant risk factors for pancreatic cancer [34]. So far, studies have failed to demonstrate the colonization and growth of $H$. pylori or inflammation triggered by $H$. pylori in the human pancreas $[35,36]$. Therefore, $H$. pylori infection may exert its effect through indirect pathophysiologic processes rather than direct stimulation. There are two plausible hypotheses explaining the role of $H$. pylori in pancreatic carcinogenesis. First, as is known, colonization of the antrum by $H$. pylori reduces the number of antral D cells, thus suppressing the production of somatostatin, which results in an increase in the secretion of secretin and pancreatic bicarbonate output. Secretin has been shown to have a positive effect on murine pancreatic growth as well as DNA synthesis in pancreatic ductal cells [37], and it can be inferred that induced ductal epithelial cell proliferation could enhance the carcinogenic effect of known carcinogens, leading to the development of pancreatic cancer. Another hypothesis is that $H$. pylori growth in the gastric corpus mucosa, leading to atrophic gastritis and hypochlorhydria, results in bacteria overgrowth and increased production of bacterially catalyzed $\mathrm{N}$-nitrosamines and transportation of these endogenous carcinogens to the host pancreas via bloodstream [38, 39].

\section{Chronic Hepatitis Virus Infection Increases the Risk of Pancreatic Cancer}

Approximately 350 million people worldwide are chronically infected by hepatitis B virus (HBV) and 180 million by hepatitis $C$ virus (HCV) [40]. China accounts for $65 \%$ of the world's HBV infective public health burden [41]. HBV and HCV are not only hepatotropic pathogens, they are also able to infect extrahepatic organs [40]. The liver and pancreas anatomically share common blood vessels and ducts, making the pancreas another potential target of hepatitis viruses [42]. In fact, by means of in situ hybridization and immunohistochemical techniques, the serological marker of present or past HBV infection, HBsAg, was detected in chronic inflammatory pancreatic acinar cells and in the pancreatic duct epithelia with pancreatic adenocarcinoma. The same was true with HCV antigen, which was also found in pancreatic acinar cells [41]. Previous studies [43, 44] suggested that both HBV and HCV can replicate in the pancreas and cause inflammation, persistent damage and subsequent possible malignant transformation. It is true that HBV and HCV are oncogenic viruses, and both are able to integrate the viral RNA or DNA into the genome of the infected cells. Viral DNA inte- 
gration into the genome of pancreatic host cells may cause deregulation of key regulators of cell control and induce tumor progression [45]. In the past few years, several studies were conducted to find the association between chronic hepatitis virus infection and the risk of pancreatic cancer. However, they have provided inconsistent results because of various factors, including study design, regional differences and so on. A study by Hong et al. [46] found no relationship between chronic HBV infection and the development of pancreatic cancer, while Ben et al. [47] reported that chronic hepatitis B and inactive hepatitis B surface antigen (HBsAg) carrier state (HBsAg positive) had a significantly increased risk of pancreatic cancer, with an adjusted OR of 1.60 (95\% CI 1.15-2.24). Noteworthy, the latest metaanalysis conducted by Xing et al. [40], including 8 case-control studies and 2 cohort studies, confirms that HBV or HCV infection is associated with an increased risk of pancreatic cancer in HBsAg- and anti-HCV-positive patients. Therefore, we speculate that a general approach based on screening and HBV vaccination programs might be a part of prevention strategies against pancreatic cancer.

\section{Oral Microbiota and Pancreatic Cancer}

Multiple observations have shown that oral microbiota overlap with the digestive tract microbiota, providing multiple avenues for dissemination in dysbiosis [1]. In a recent retrospective case-control study, oral bacteria measures in saliva were associated with pancreatic cancer [48]. Michaud et al. [1] found increased levels of antibodies against specific commensal oral bacteria, which can inhibit growth of pathogenic bacteria and might reduce the risk of pancreatic cancer. The possible mechanisms include Toll-like receptor signaling, nitrosamine exposure and increase of systemic inflammation [49]. Research on the oral microbiome and pancreatic cancer may open new opportunities to develop biomarkers to identify high-risk individuals.

\section{Summary}

Microbiota now appear to influence the host at nearly every level and in every organ system, highlighting our interdependence and co-evolution. Accumulating evidence indicates that there is a close relationship between pathogenic microorganisms and pancreatic cancer. More research in this area will likely lead to a better understanding of this highly fatal cancer and may bring insight into mechanisms that may result in new opportunities for early detection and/or treatment development.

\section{References}

1 Michaud DS, Izard J, Wilhelm-Benartzi CS, et al: Plasma antibodies to oral bacteria and risk of pancreatic cancer in a large European prospective cohort study. Gut 2013;62:1764-1770.

2 Qin J, Li R, Raes J, et al: A human gut microbial gene catalogue established by metagenomic sequencing. Nature 2010;464:59-65.

3 Kau AL, Ahern PP, Griffin NW, Goodman AL, Gordon JI: Human nutrition, the gut microbiome and the immune system. Nature 2011;474:327-336.

4 Sekirov I, Russell SL, Antunes LC, Finlay BB: Gut microbiota in health and disease. Physiol Rev 2010;90:859904.

5 McFarland LV: Antibiotic-associated diarrhea: epidemiology, trends and treatment. Future Microbiol 2008;3: 563-578.

6 Iapichino G, Callegari ML, Marzorati S, et al: Impact of antibiotics on the gut microbiota of critically ill patients. J Med Microbiol 2008;57:1007-1014. 
Wang and Li: Pathogenic Microorganisms and Pancreatic Cancer

7 Spiller R, Garsed K: Postinfectious irritable bowel syndrome. Gastroenterology 2009;136:1979-1988.

8 Abraham C, Cho JH: Inflammatory bowel disease. N Engl J Med 2009;361:2066-2078.

9 Sartor RB: Genetics and environmental interactions shape the intestinal microbiome to promote inflammatory bowel disease versus mucosal homeostasis. Gastroenterology 2010;139:1816-1819.

10 Correa P, Houghton J: Carcinogenesis of Helicobacter pylori. Gastroenterology 2007;133:659-672.

11 Zambirinis CP, Pushalkar S, Saxena D, Miller G: Pancreatic cancer, inflammation, and microbiome. Cancer J 2014;20:195-202.

12 Ciorba MA: A gastroenterologist's guide to probiotics. Clin Gastroenterol Hepatol 2012;10:960-968.

13 Ley RE, Turnbaugh PJ, Klein S, Gordon JI: Microbial ecology: human gut microbes associated with obesity. Nature 2006;444:1022-1023.

14 Liu CH, Yang XQ, Liu CH, He Y, Wang LJ: Allergic airway response associated with the intestinal microflora disruption induced by antibiotic therapy (in Chinese). Zhonghua Er Ke Za Zhi 2007;45:450-454.

15 Schwartz RF, Neu J, Schatz D, Atkinson MA, Wasserfall C: Comment on: Brugman S et al. (2006): Antibiotic treatment partially protects against type 1 diabetes in the Bio-Breeding diabetes-prone rat. Is the gut flora involved in the development of type 1 diabetes? Diabetologia 49:2105-2108. Diabetologia 2007;50:220-221.

16 Finegold SM, Molitoris D, Song Y, et al: Gastrointestinal microflora studies in late-onset autism. Clin Infect Dis 2002;35(suppl 1):S6-S16.

17 Raimondi S, Maisonneuve P, Lowenfels AB: Epidemiology of pancreatic cancer: an overview. Nat Rev Gastroenterol Hepatol 2009;6:699-708.

18 Yadav D, Lowenfels AB: The epidemiology of pancreatitis and pancreatic cancer. Gastroenterology 2013;144: 1252-1261.

19 Ma C, Jiang YX, Liu SZ, Quan PL, Sun XB, Zheng RS, Zhang SW, Chen WQ: Trend and prediction on the incidence of pancreatic cancer in China (in Chinese). Zhonghua Liu Xing Bing Xue Za Zhi 2013;34:160-163.

20 Li D, Xie K, Wolff R, Abbruzzese JL: Pancreatic cancer. Lancet 2004;363:1049-1057.

21 Maisonneuve P, Lowenfels AB: Risk factors for pancreatic cancer: a summary review of meta-analytical studies. Int J Epidemiol 2015;44:186-198.

22 Iodice S, Gandini S, Maisonneuve P, Lowenfels AB: Tobacco and the risk of pancreatic cancer: a review and meta-analysis. Langenbecks Arch Surg 2008;393:535-545.

23 Xu PHQ, Liu CXF, Shao FZC, Lei L: Risk factors for pancreatic cancer: a case-control study. Tumor 2011;31: 653-657.

24 Larsen N, Vogensen FK, van den Berg FW, et al: Gut microbiota in human adults with type 2 diabetes differs from non-diabetic adults. PLoS One 2010;5:e9085.

25 Qin J, Li Y, Cai Z, et al: A metagenome-wide association study of gut microbiota in type 2 diabetes. Nature 2012; 490:55-60.

26 Han JL, Lin HL: Intestinal microbiota and type 2 diabetes: from mechanism insights to therapeutic perspective. World J Gastroenterol 2014;20:17737-17745.

27 Shen J, Obin MS, Zhao L: The gut microbiota, obesity and insulin resistance. Mol Aspects Med 2013;34:39-58.

28 Furet JP, Kong LC, Tap J, et al: Differential adaptation of human gut microbiota to bariatric surgery-induced weight loss: links with metabolic and low-grade inflammation markers. Diabetes 2010;59:3049-3057.

29 Armougom F, Henry M, Vialettes B, Raccah D, Raoult D: Monitoring bacterial community of human gut microbiota reveals an increase in Lactobacillus in obese patients and Methanogens in anorexic patients. PLoS One 2009; 4:e7125.

30 Million M, Maraninchi M, Henry M, et al: Obesity-associated gut microbiota is enriched in Lactobacillus reuteri and depleted in Bifidobacterium animalis and Methanobrevibacter smithii. Int J Obes (Lond) 2012;36:817-825.

31 Backhed F, Manchester JK, Semenkovich CF, Gordon JI: Mechanisms underlying the resistance to diet-induced obesity in germ-free mice. Proc Natl Acad Sci USA 2007;104:979-984.

32 Brenner H, Rothenbacher D, Arndt V: Epidemiology of stomach cancer. Methods Mol Biol 2009;472:467-477.

33 Stolzenberg-Solomon RZ, Blaser MJ, Limburg PJ, et al: Helicobacter pylori seropositivity as a risk factor for pancreatic cancer. J Natl Cancer Inst 2001;93:937-941.

34 Gawin A, Wex T, Lawniczak M, Malfertheiner P, Starzynska T: Helicobacter pylori infection in pancreatic cancer (in Polish). Pol Merkur Lekarski 2012;32:103-107.

35 Risch HA: Pancreatic cancer: Helicobacter pylori colonization, N-nitrosamine exposures, and ABO blood group. Mol Carcinog 2012;51:109-118.

36 Nilsson HO, Stenram U, Ihse I, Wadstrom T: Helicobacter species ribosomal DNA in the pancreas, stomach and duodenum of pancreatic cancer patients. World J Gastroenterol 2006;12:3038-3043.

37 Bulajic M, Panic N, Lohr JM: Helicobacter pylori and pancreatic diseases. World J Gastrointest Pathophysiol 2014;5:380-383.

38 Kokkinakis DM, Reddy MK, Norgle JR, Baskaran K: Metabolism and activation of pancreas specific nitrosamines by pancreatic ductal cells in culture. Carcinogenesis 1993;14:1705-1709.

39 Houben GM, Stockbrugger RW: Bacteria in the aetio-pathogenesis of gastric cancer: a review. Scand J Gastroenterol Suppl 1995;212:13-18.

40 Xing S, Li ZW, Tian YF, Zhang LM, Li MQ, Zhou P: Chronic hepatitis virus infection increases the risk of pancreatic cancer: a meta-analysis. Hepatobiliary Pancreat Dis Int 2013;12:575-583.

$41 \mathrm{Xu} \mathrm{JH}, \mathrm{Fu}$ JJ, Wang XL, Zhu JY, Ye XH, Chen SD: Hepatitis B or C viral infection and risk of pancreatic cancer: a meta-analysis of observational studies. World J Gastroenterol 2013;19:4234-4241. 
42 Wang DS, Chen DL, Ren C, et al: ABO blood group, hepatitis B viral infection and risk of pancreatic cancer. Int J Cancer 2012;131:461-468.

43 Katakura Y, Yotsuyanagi H, Hashizume K, et al: Pancreatic involvement in chronic viral hepatitis. World J Gastroenterol 2005;11:3508-3513.

44 Taranto D, Carrato A, Romano M, Maio G, Izzo CM, Del Vecchio Blanco C: Mild pancreatic damage in acute viral hepatitis. Digestion 1989;42:93-97.

45 Hassan MM, Li D, El-Deeb AS, et al: Association between hepatitis B virus and pancreatic cancer. J Clin Oncol 2008;26:4557-4562.

46 Hong SG, Kim JH, Lee YS, et al: The relationship between hepatitis B virus infection and the incidence of pancreatic cancer: a retrospective case-control study (in Korean). Korean J Hepatol 2010;16:49-56.

47 Ben Q, Li Z, Liu C, et al: Hepatitis B virus status and risk of pancreatic ductal adenocarcinoma: a case-control study from China. Pancreas 2012;41:435-440.

48 Farrell JJ, Zhang L, Zhou H, et al: Variations of oral microbiota are associated with pancreatic diseases including pancreatic cancer. Gut 2012;61:582-588.

49 Michaud DS, Izard J: Microbiota, oral microbiome, and pancreatic cancer. Cancer J 2014;20:203-206. 